\title{
Opportunities and Threats: The Necessity of Research in Dealing with the Rapid Spread of Coronavirus disease 2019 (COVID-19) in Iran
}

\author{
Abdorreza Naser Moghadasi (iD) ${ }^{1, *}$ \\ ${ }^{1}$ Multiple Sclerosis Research Center, Neuroscience institute, Tehran University of Medical Sciences, Tehran, Iran \\ "Corresponding author: Multiple Sclerosis Research Center, Neuroscience institute, Tehran University of Medical Sciences, Tehran, Iran. Email: \\ abdorrezamoghadasi@gmail.com
}

Received 2020 March 17; Accepted 2020 March 25.

Keywords: Opportunities, Threats, COVID-19, Iran

Although statistics obtained from different sources about the prevalence of coronavirus in Iran are different and sometimes contradictory, they all indicate its rapid spread in various regions of the country (1). At present, all sectors of the Iranian medical care system are involved in the outbreak of coronavirus disease 2019 (COVID-19), and the first objective of all efforts made is to contain the disease, treat infected patients, and reduce the mortality rate. However, more health care challenges are beginning to emerge as the virus spreads wider. The primary purpose in managing all epidemics is to prevent the disease. As a general rule, it is always assumed that individuals with a weak immune system are more likely to develop these diseases and if they do so, they will experience more severe conditions. Children, the elderly, pregnant women, and those having an underlying disease or receiving immunosuppressive drugs have been usually regarded as high-risk groups. As a neurologist, these days I am heavily involved in the treatment of patients, each of whom may be at risk of COVID-19 and its complications. I mainly visit multiple sclerosis (MS) patients, the vast majority of whom receive drugs such as Tysabri, Rituximab, Ocrelizumab, Dimethyl fumarate, and Fingolimod. Each of the mentioned drugs can weaken the patients' immune system (2). Moreover, these patients are also highly susceptible to MS attacks due to the presence of various recent stressors in Iran. Although a large proportion of different treatment guidelines have always been considered for the high-risk groups, there are no national guidelines on how to deal with MS patients in the present critical situation. These days, other colleagues dealing with MS patients repeatedly ask similar questions regarding whether they should continue the previous routine medications for MS patients; whether the continuation of these medications could make this group of patients susceptible to COVID-19; and whether corticosteroids should be used for the treatment in case of MS attacks, or if plasmapheresis or intravenous immunoglobulin (IVIG) is a preferred treatment. There are no definitive answers to these significant questions, which could potentially affect the life of an MS patient. It should be taken into consideration that exposure to a coronavirus is not the first exposure to such epidemics in Iran. Although the prevalence of the previous outbreaks and epidemics with viruses such as H1N1 or Middle East Respiratory Syndromerelated coronavirus (MERS-CoV) may not be comparable to that of COVID-19, the potential complications were equally significant for the infected patients by viruses such as H1N1 or MERS-CoV. Unfortunately, we do not have any statistics on the involved number of MS patients treated with various types of drugs during those epidemics. As far as the researcher has assessed, only one study has addressed the neurological complications induced by the H1N1 virus in Iran (3). The mentioned study has not answered the above questions in any way. Earlier epidemics, however, could have provided a valuable opportunity for research studies and provision of appropriate guidelines for broader and more severe conditions similar to those in the present days of Iran. Previous research opportunities in Iran have been largely ignored, which should not repeat in this recent epidemic. Of course, the problem that neurologists currently have with the treatment of MS is not limited to them. Many other physicians working in other fields such as oncology, rheumatology, nephrology and dermatology face similar challenges. Answering these questions requires a rigorous and systematic research plan which has not been designed in Iran due to various medical shortcomings. Although the spread of the coronavirus is a major threat to the health of the Iranian community, physicians should consider it 
an opportunity by performing extensive fieldwork and research studies; it will not only enhance their knowledge but can also be used to offer appropriate guidelines to act properly against similar future threats.

\section{Footnotes}

Conflict of Interests: The author declares there is no conflict of interest.

Funding/Support: This study is not funded.

\section{References}

1. World Health Organization. Coronavirus disease 2019 (COVID-19). 2020. Available from: https://www.who.int/docs/default-source/ coronaviruse/situation-reports.

2. Luna G, Alping P, Burman J, Fink K, Fogdell-Hahn A, Gunnarsson M, et al. Infection risks among patients with multiple sclerosis treated with fingolimod, natalizumab, rituximab, and injectable therapies. JAMA Neurol. 2019. doi: 10.1001/jamaneurol.2019.3365. [PubMed: 31589278]. [PubMed Central: PMC6784753].

3. Asadi-Pooya AA, Yaghoubi E, Nikseresht A, Moghadami M, Honarvar $B$. The neurological manifestations of H1N1 influenza infection; diag nostic challenges and recommendations. Iran J Med Sci. 2011;36(1):369. [PubMed: 23365476]. [PubMed Central: PMC3559119]. 